\title{
Solid tumors versus mixed tumors with a ground-glass opacity component in patients with clinical stage IA lung adenocarcinoma: Prognostic comparison using high-resolution computed tomography findings
}

\author{
Yasuhiro Tsutani, MD, PhD, ${ }^{\text {a }}$ Yoshihiro Miyata, MD, PhD, ${ }^{\mathrm{a}}$ Takeharu Yamanaka, PhD, ${ }^{\mathrm{b}}$ \\ Haruhiko Nakayama, MD, PhD, ${ }^{\mathrm{c}}$ Sakae Okumura, MD, PhD, ${ }^{\mathrm{d}}$ Shuji Adachi, MD, PhD, ${ }^{\mathrm{e}}$ \\ Masahiro Yoshimura, MD, $\mathrm{PhD},{ }^{\mathrm{f}}$ and Morihito Okada, $\mathrm{MD}, \mathrm{PhD}^{\mathrm{a}}$
}

Objective: This study aimed to compare malignant behavior and prognosis between solid tumors and mixed tumors with a ground-glass opacity component on high-resolution computed tomography.

Methods: We examined 436 of 502 consecutive patients with clinical stage IA adenocarcinoma who had undergone
preoperative high-resolution computed tomography and F-18-fluorodeoxyglucose positron emission tomography/
computed tomography; 66 patients with tumors with pure ground-glass opacity components were excluded. Tumor
type (solid, $n=137$; mixed, $\mathrm{n}=299$ ) and surgical results were analyzed for all patients and their matched pairs.

Results: In all patients, solid tumors showed a significantly greater association $(P<.001)$ with lymphatic, vascular, and pleural invasion and lymph node metastasis compared with mixed tumors. The disease-free survival was also worse in patients with solid tumors $(P=.0006)$. Analysis of 97 pairs matched for solid component size confirmed that solid tumors were significantly associated with lymphatic, vascular, and pleural invasion ( $P=.008, P=.029, P=.003$, respectively) and poor prognosis. When maximum standardized uptake value and solid component size were matched $(\mathrm{n}=79)$, the differences in pathologic prognostic parameters and disease-free survivals between patients with solid and mixed tumors disappeared.

Conclusions: Solid tumors exhibit more malignant behavior and have a poorer prognosis compared with mixed tumors, even when the solid component size is the same in both tumor types. However, differences in malignant behavior can be identified using maximum standardized uptake values determined by F-18-fluorodeoxyglucose positron emission tomography/computed tomography. (J Thorac Cardiovasc Surg 2013;146:17-23)

Earn CME credits at

http://cme.ctsnetjournals.org

The recent development of high-resolution computed tomography (HRCT) and low-dose computed tomography (CT) screening has improved the detection of small lung cancers, especially lung adenocarcinomas. ${ }^{1-3}$ These often contain a nonsolid component that presents as a ground-

\footnotetext{
From the Department of Surgical Oncology, ${ }^{a}$ Hiroshima University, Hiroshima, Japan; Research Center for Innovative Oncology, ${ }^{\mathrm{b}}$ National Cancer Center Hospital East, Kashiwa, Japan; Department of Thoracic Surgery, ${ }^{\mathrm{c}}$ Kanagawa Cancer Center, Yokohama, Japan; Department of Thoracic Surgery, ${ }^{\mathrm{d}}$ Cancer Institute Hospital, Tokyo, Japan; and Departments of Radiology ${ }^{\mathrm{e}}$ and Thoracic Surgery, ${ }^{\mathrm{f}}$ Hyogo Cancer Center, Akashi, Japan.

Disclosures: Authors have nothing to disclose with regard to commercial support.

Received for publication Jan 23, 2012; revisions received Sept 14, 2012; accepted for publication Nov 6, 2012; available ahead of print Dec 14, 2012.

Address for reprints: Morihito Okada, MD, PhD, Department of Surgical Oncology, Research Institute for Radiation Biology and Medicine, Hiroshima University, 1-2-

3-Kasumi, Minami-ku, Hiroshima City, Hiroshima 734-0037, Japan (E-mail: morihito@hiroshima-u.ac.jp).

$0022-5223 / \$ 36.00$

Copyright (C) 2013 by The American Association for Thoracic Surgery

http://dx.doi.org/10.1016/j.jtcvs.2012.11.019
}

glass opacity (GGO) on HRCT and is closely associated with bronchioloalveolar carcinoma. ${ }^{4,5}$ We have previously reported the benefits of comparing solid component size (the maximum dimension of the solid component excluding GGO) on HRCT with whole tumor size for predicting the pathologic invasiveness of tumors or the prognosis of clinical stage IA lung adenocarcinomas. ${ }^{6}$ It remains unclear whether GGO-containing tumors have the same malignant behavior and prognosis as pure solid tumors after matching for solid component size.

Whether or not differences exist in malignant behavior between pure solid tumors and mixed tumors with a GGO component on HRCT remains controversial. Therefore, we used HRCT to compare malignant behavior, including lymphatic, vascular, and pleural invasion, and prognosis between solid tumors and mixed tumors having a GGO component in patients with clinical stage IA lung adenocarcinoma.

\section{PATIENTS AND METHODS}

Between August 1, 2005, and December 31, 2009, we enrolled 502 patients with clinical T1N0M0 stage IA lung adenocarcinoma who were admitted to 1 of the following 4 institutions: Hiroshima University, Kanagawa Cancer Center, Cancer Institute Hospital, and Hyogo Cancer Center. HRCT and F-18-fluorodeoxyglucose positron emission tomography/CT 


\section{Abbreviations and Acronyms \\ CT = computed tomography \\ DFS = disease-free survival \\ FDG- $\quad=$ F-18-fluorodeoxyglucose positron \\ PET emission tomography \\ FOV = field of view \\ GGO = ground-glass opacity \\ HRCT = high-resolution computed tomography \\ SUV $=$ standardized uptake value \\ SUVmax $=$ maximum standardized uptake value}

(FDG-PET/CT) followed by curative R0 resection were performed in all patients, who were staged according to the seventh edition of the TNM classification of malignant tumors. ${ }^{7}$ Mediastinoscopy and endobronchial ultrasonography were not routinely performed because HRCT revealed no swelling of mediastinal or hilar lymph nodes and FDG-PET showed no accumulation in these lymph nodes in all patients. Sublobar resections (segmentectomy or wedge resection) were performed if the tumor mainly comprised a GGO component or had no lymph node metastasis on intraoperative assessment. Tumors with pure GGO were excluded from the analyses because they are noninvasive and have an extremely good prognosis. ${ }^{8,9}$ We obtained appropriate approval for this multicenter study from the institutional review board of each institution, which waived the requirement for informed consent from individual patients because this was a retrospective review of medical records from a prospective database.

\section{High-Resolution Computed Tomography}

Chest images were obtained using 16-row multidetector CT independently of subsequent FDG-PET/CT examinations. High-resolution images of the tumors were acquired using the following parameters: $120 \mathrm{kVp} ; 200$ $\mathrm{mA}$; section thickness, 1 to $2 \mathrm{~mm}$; pixel resolution, $512 \times 512$; scanning time, 0.5 to 1 seconds; a high spatial reconstruction algorithm with a 20 -cm field of view (FOV); and mediastinal (level, $40 \mathrm{HU}$; width, 400 $\mathrm{HU}$ ) and lung (level, $-600 \mathrm{HU}$; width, $1600 \mathrm{HU}$ ) window settings. GGO was defined as a misty increase in lung attenuation that did not obscure underlying vascular markings. We defined solid component size as the maximum dimension of the solid component in the lung windows after excluding the GGO component. ${ }^{6}$ Solid tumors were defined as pure solid tumors without a GGO component, whereas mixed tumors were defined as tumors with a GGO component regardless of the GGO proportion.

\section{F-18-Fluorodeoxyglucose Positron Emission Tomography/Computed Tomography}

Patients were instructed to fast for more than 4 hours before intravenous injection of 74 to $370 \mathrm{MBq}$ of FDG. After injection, they were instructed to relax for at least 1 hour before FDG-PET/CT scanning. Blood glucose was calculated before tracer injection to confirm a level of less than 150 $\mathrm{mg} / \mathrm{dL}{ }^{10}$ Patients with blood glucose values $150 \mathrm{mg} / \mathrm{dL}$ or greater were excluded from PET/CT image acquisition. Images were obtained using Discovery ST (GE Healthcare, Little Chalfont, UK), Aquiduo (Toshiba Medical Systems Corporation, Tochigi, Japan), or Biograph Sensation16 (Siemens Healthcare, Erlangen, Germany) integrated PET/CT scanners. Low-dose, unenhanced CT images of 2- to 4-mm section thickness for attenuation correction and localization of lesions identified by PET were obtained from the head to the pelvic floor of each patient using a standard protocol. Immediately after CT, PET covered the identical axial FOV for 2 to 4 minutes per table position depending on the condition of the patient and scanner performance. All PET images with a 50-cm FOV were reconstructed using an iterative algorithm with CT-derived attenuation correction. Variations in standardized uptake values (SUVs) among institutions were minimized using an anthropomorphic body phantom. A calibration factor was obtained by dividing the actual SUV by the gauged mean SUV in the phantom background to decrease interinstitutional SUV inconsistencies; the final SUV used is referred to as the revised maximum SUV (SUVmax). ${ }^{1,12}$ Adjustment of interinstitutional variability in SUV narrowed the range from 0.89 to 1.24 to 0.97 to 1.18 when the SUVmax ratio was expressed as the SUVmax reported by each institute relative to the SUVmax reported by the control institute.

\section{Follow-up Evaluation}

All patients who underwent lung resection were followed up from the day of surgery. Postoperative follow-up procedures, including physical examination and chest roentgenography every 3 months and chest and abdominal CT examinations every 6 months, were performed for the first 2 years. Thereafter, physical examination and chest roentgenography were performed every 6 months, whereas chest CT examination was performed every year. Recurrence was determined by radiographic features or histologic evidence.

\section{Statistical Analysis}

Data are presented as numbers ( $\%$ ) or mean \pm standard deviation unless otherwise stated. Frequencies were compared using the chi-square test for categoric variables, and the Fisher exact test was applied to small samples in all cohort patients. McNemar tests were used for analyses of matchedpair patients. Mann-Whitney $U$ tests and $t$ tests were used to compare continuous variables in all cohort patients. Wilcoxon tests were used for analyses of matched-pair patients. Disease-free survival (DFS) was defined as the time from the date of surgery until the first event (relapse or death from any cause) or last follow-up. The duration of DFS was analyzed using the Kaplan-Meier method. Differences in DFS were assessed using the logrank test. We applied matching to balance the assignment of the included patients and correct for tumor type (solid or mixed), which confounded survival. The variables were solid component size or SUVmax. Solid and mixed tumor pairs with an equivalent solid component size or SUVmax were selected by a 1-to- 1 match. All 436 patients were pooled and sorted in ascending order according to their solid component size or SUVmax. The selection process began from the first 2 cases with the lowest solid component size or SUVmax. If 1 case exhibited a solid tumor and the other case exhibited a mixed tumor, both were selected as a matched pair. If this was not the case, then 4 cases were included. In the same way, solid and mixed tumors were matched by their solid component size or SUVmax in $1: 1,2: 2,3: 3$, or $4: 4$ blocks. A patient who did not have a suitable match within the acceptable rank range was excluded from further analysis, and the matching process moved down the sort list until all possible matched pairs were included. The selected patients formed well-matched 1:1 pairs in both groups. Data were analyzed using the Statistical Package for the Social Sciences (v 10.5; SPSS Inc, Chicago, Ill).

\section{RESULTS}

Of the 502 patients, 66 who had tumors with pure GGO components were excluded; the remaining 436 patients were included in this analysis. Of the 436 study patients, 137 had solid tumors and 299 had mixed tumors. The mean follow-up period after surgery was $20.2 \pm 12.5$ months, during which the disease recurred in 29 patients $(6.7 \%)$. The mean follow-up period was similar for solid and mixed tumors $(21.4 \pm 12.8$ months and $19.7 \pm 12.4$ months, respectively, $P=.235$ ). Of the 29 cases of recurrence, $9(2.1 \%)$ were local (including mediastinal lymph node metastasis), $3(0.7 \%)$ were local and distant, and 17 
(3.9\%) were distant. Age, sex, and whole tumor size on HRCT were not significantly different between patients with solid and mixed tumors. Solid tumors were significantly correlated with a large solid component size, a high SUVmax, and the presence of lymphatic, vascular, and pleural invasion and lymph node metastasis $(P<.001, P<.001, P<.001, P<.001, P<.001$, $P=.001$, respectively; Table 1 ).

Local recurrence occurred in 5 patients $(3.6 \%)$ with solid tumors ( 1 involving the bronchial stump and 4 involving the mediastinal lymph nodes) and 4 patients $(1.3 \%)$ with mixed tumors ( 1 involving the residual lung after segmentectomy and 3 involving the mediastinal lymph nodes). A significant difference in DFS was identified between patients with solid tumors $(\mathrm{n}=137$; 2-year DFS, 83.1\%) and those with mixed tumors $(\mathrm{n}=299$; 2-year DFS, 94.2\%; $P=.0006$; Figure $1, A$ ).

After matching for solid component size, there were 97 well-matched solid and mixed tumor pairs. Significant differences were identified in whole tumor size, SUVmax, and lymphatic, vascular, and pleural invasion between the 2 tumor types $(P<.001, P<.001, P=.008, P=.029, P=.003$, respectively, Table 2 ). Solid tumors were significantly correlated with a small whole tumor size, a high SUVmax, and the presence of pathologic invasiveness.

Furthermore, a difference in DFS was identified between patients with solid tumors $(\mathrm{n}=97$; 2-year DFS, $83.5 \%)$ and

TABLE 1. Comparison of solid and mixed tumor characteristics in all cohort patients

\begin{tabular}{|c|c|c|c|}
\hline & $\begin{array}{l}\text { Solid tumors } \\
\quad(n=137)\end{array}$ & $\begin{array}{l}\text { Mixed tumors } \\
\quad(n=299)\end{array}$ & $\boldsymbol{P}$ \\
\hline Age $(y)$ & $65.5 \pm 10.5$ & $65.7 \pm 8.8$ & .85 \\
\hline Sex & & & .12 \\
\hline Male & $71(51.8 \%)$ & $130(43.5 \%)$ & \\
\hline Female & $66(48.2 \%)$ & $169(56.5 \%)$ & \\
\hline Whole tumor size $(\mathrm{cm})$ & $2.1 \pm 0.6$ & $2.0 \pm 0.6$ & .69 \\
\hline Solid component size $(\mathrm{cm})$ & $2.1 \pm 0.6$ & $1.1 \pm 0.7$ & $<.001$ \\
\hline SUVmax & $4.9 \pm 3.3$ & $2.6 \pm 2.9$ & $<.001$ \\
\hline Lymphatic invasion & & & $<.001$ \\
\hline Negative & $89(65.0 \%)$ & $270(90.3 \%)$ & \\
\hline Positive & $48(35.0 \%)$ & $29(9.7 \%)$ & \\
\hline Vascular invasion & & & $<.001$ \\
\hline Negative & $79(57.7 \%)$ & $264(88.3 \%)$ & \\
\hline Positive & $58(42.3 \%)$ & $35(11.7 \%)$ & \\
\hline Pleural invasion & & & $<.001$ \\
\hline Negative & $100(73.0 \%)$ & $278(93.0 \%)$ & \\
\hline Positive & $37(27.0 \%)$ & $21(7.0 \%)$ & \\
\hline Lymph node metastasis & & & $<.001$ \\
\hline Negative & $114(83.2 \%)$ & $284(95.0 \%)$ & \\
\hline Positive & $23(16.8 \%)$ & $15(5.0 \%)$ & \\
\hline Procedure & & & .001 \\
\hline Lobectomy & $111(81.0 \%)$ & $190(63.5 \%)$ & \\
\hline Segmentectomy & $9(6.6 \%)$ & $48(16.1 \%)$ & \\
\hline Wedge resection & $17(12.4 \%)$ & $61(20.4 \%)$ & \\
\hline
\end{tabular}

SUVmax, Maximum standardized uptake value. those with mixed tumors $(\mathrm{n}=97$; 2-year DFS, 91.8\%; Figure 1, B) after matching for solid component size.

After matching for SUVmax, there were 96 well-matched solid and mixed tumor pairs. No significant differences in clinical characteristics, except for solid component size, were found between the 2 tumor types (Table 3 ).

A difference in DFS was identified between patients with solid tumors ( $\mathrm{n}=96$; 2-year DFS, $87.1 \%$ ) and those with mixed tumors $(\mathrm{n}=96$; 2-year DFS, 90.4\%; Figure $1, C)$ after matching for SUVmax.

After matching for solid component size and SUVmax, there were 79 well-matched solid and mixed tumor pairs. No significant differences in clinical characteristics, except for whole tumor size, were found between the 2 tumor types (Table 4).

Furthermore, there was no difference in DFS between patients with solid tumors $(\mathrm{n}=79 ; 2$-year DFS, 87.0\%) and patients with mixed tumors $(\mathrm{n}=79$; 2-year DFS, $83.9 \%$; Figure $1, D$ ) after matching for solid component size and SUVmax.

Figure 2 shows examples of solid and mixed tumors with the same solid component size $(1.0 \mathrm{~cm})$. Regardless of tumor type, tumors with low SUVmax were not associated with lymphatic invasion, whereas those with high SUVmax were.

\section{DISCUSSION}

The present study demonstrated, as expected, that solid tumors were associated with highly malignant variables, such as large solid component size, high SUVmax, and lymphatic, vascular, and pleural invasion and lymph node metastasis in all cohort patients. In addition, patients with solid tumors had worse DFS than those with mixed tumors. A retrospective study has previously shown that pure solid tumors have malignant potential with nodal or pleural involvement and worse DFS compared with predominantly solid tumors with a GGO component. ${ }^{13}$ Other studies have also revealed that tumors with a predominant GGO component are less invasive and have a more favorable prognosis in patients with clinical stage IA lung adenocarcinomas. ${ }^{4,8,14}$ Our study is consistent with these findings.

With regard to the tumor size on HRCT, solid component size is more useful than whole tumor size for predicting pathologic invasiveness and prognosis. In our previous study, solid component size was found to have a higher predictive value for lymphatic, vascular, and pleural invasion compared with whole tumor size; furthermore, solid component size was an independent prognostic factor for DFS. ${ }^{6}$ It was not clear whether mixed tumors and solid tumors have similar malignant behaviors and prognoses when both have the same solid component size on HRCT. Therefore, we conducted a matched analysis to compare solid and mixed tumors after matching for solid component size in both tumor types. Even after matching for solid component

西



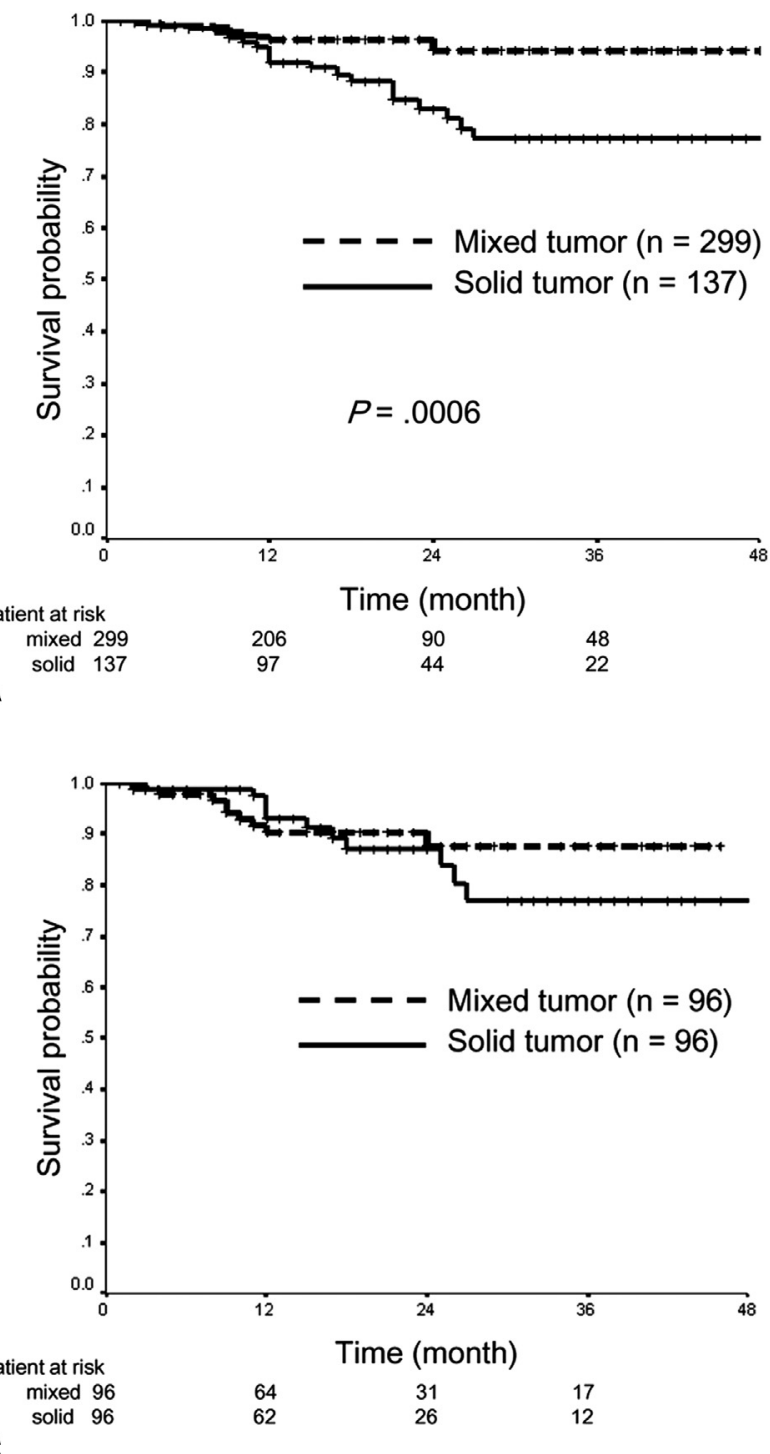

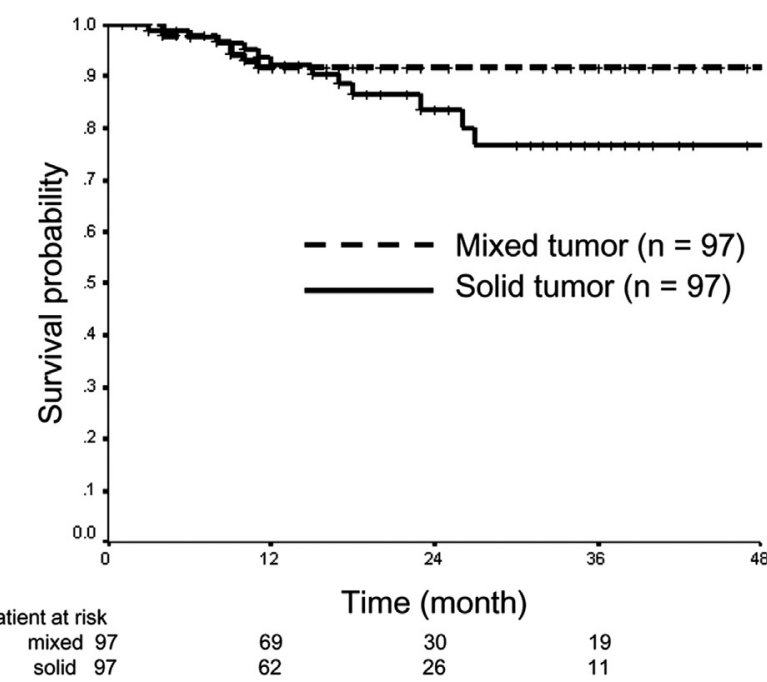

B

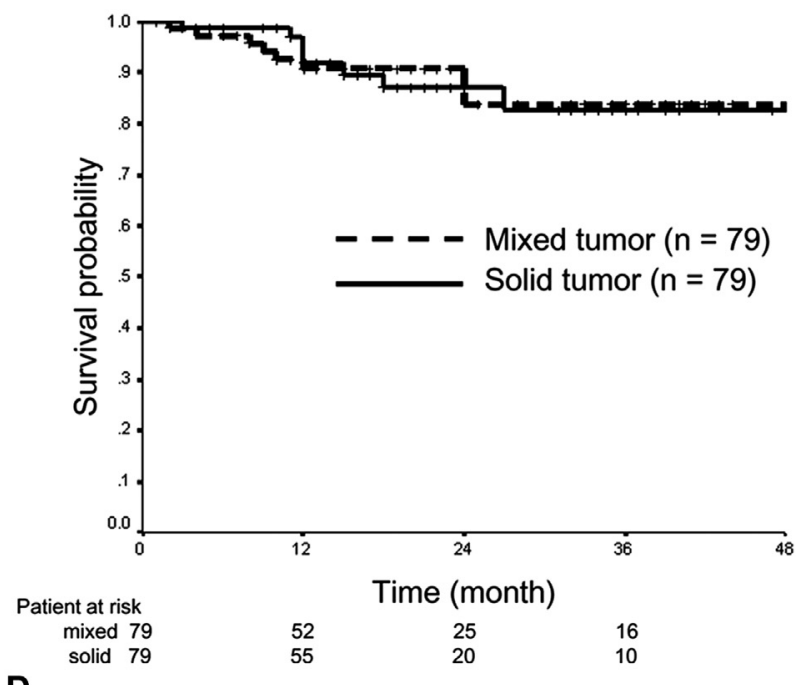

D

FIGURE 1. DFS curves of patients according to tumor type on HRCT. A, In all cohort patients, 2-year DFS of $94.2 \%$ (mean DFS of 47 months; $95 \%$ confidence interval [CI], 46-48 months) and 83.1\% (mean DFS of 42 months; 95\% CI, 39-45 months) were identified for mixed and solid tumors, respectively $(P=.0006)$. B, In patients matched for solid component size, 2-year DFS of $91.8 \%$ (mean DFS of 46 months; $95 \%$ CI, $43-48$ months) and $83.5 \%$ (mean DFS of 42 months; $95 \%$ CI, 38-45 months) were identified for mixed and solid tumors, respectively. C, In patients matched for SUVmax, 2-year DFS of $90.4 \%$ (mean DFS of 42 months; 95\% CI, 39-44 months) and 87.1\% (mean DFS of 42 months; 95\% CI, 38-46 months) were detected for mixed and solid tumors, respectively. D, In patients matched for solid component size and SUVmax, 2-year DFS of 83.9\% (mean DFS of 43 months; $95 \%$ CI, 4047 months) and $87.0 \%$ (mean DFS of 43 months; $95 \%$ CI, 40-47 months) were detected for mixed and solid tumors, respectively.

size in both tumor types on HRCT, solid tumors were more frequently correlated with high SUVmax and malignant behavior compared with mixed tumors. In addition, the DFS of patients with solid tumors was worse than that of patients with mixed tumors. This means that solid tumors have more malignant potential than mixed tumors even if both tumor types have the same solid component size on HRCT. This is a new finding. SUVmax on PET/CT is reported to be a predictor of malignant behavior and prognosis in cases of lung adenocarcinomas. ${ }^{6,11,12,15-17}$ SUVmax on PET/CT is a preoperative factor, whereas lymphatic, vascular, and pleural invasion are postoperative factors. We have previously reported that SUVmax is a significant predictor of malignant behavior. ${ }^{6,11,12,16,17}$

We experimentally performed a matched analysis to compare solid and mixed tumors after matching for SUVmax. In this matched model, solid tumors and mixed tumors had similar clinical characteristics except solid component size, but there seemed to be a difference in DFS. Although both tumor types have the same SUVmax, solid tumors seem to have a worse potential than mixed tumors. 
TABLE 2. Comparison of solid and mixed tumor characteristics in patients matched for solid component size

\begin{tabular}{|c|c|c|c|}
\hline & $\begin{array}{l}\text { Solid tumors } \\
\quad(n=97)\end{array}$ & $\begin{array}{l}\text { Mixed tumors } \\
\quad(\mathbf{n}=97)\end{array}$ & $\boldsymbol{P}$ \\
\hline Age (y) & $64.9 \pm 10.4$ & $66.1 \pm 10.0$ & .63 \\
\hline Sex & & & .054 \\
\hline Male & $50(51.5 \%)$ & $36(37.1 \%)$ & \\
\hline Female & $47(48.5 \%)$ & $61(62.9 \%)$ & \\
\hline Whole tumor size $(\mathrm{cm})$ & $1.8 \pm 0.5$ & $2.3 \pm 0.5$ & $<.001$ \\
\hline Solid component size $(\mathrm{cm})$ & $1.8 \pm 0.5$ & $1.8 \pm 0.5$ & N/A \\
\hline SUVmax & $4.8 \pm 3.4$ & $3.0 \pm 2.5$ & $<.001$ \\
\hline Lymphatic invasion & & & .008 \\
\hline Negative & $63(64.9 \%)$ & $81(83.5 \%)$ & \\
\hline Positive & $34(35.1 \%)$ & $16(16.5 \%)$ & \\
\hline Vascular invasion & & & .029 \\
\hline Negative & $62(63.9 \%)$ & $76(78.4 \%)$ & \\
\hline Positive & $35(36.1 \%)$ & $21(21.6 \%)$ & \\
\hline Pleural invasion & & & .003 \\
\hline Negative & $71(73.2 \%)$ & $88(90.1 \%)$ & \\
\hline Positive & $26(26.8 \%)$ & $9(9.9 \%)$ & \\
\hline Lymph node metastasis & & & .13 \\
\hline Negative & $82(84.5 \%)$ & $90(92.8 \%)$ & \\
\hline Positive & $15(15.5 \%)$ & $7(7.2 \%)$ & \\
\hline Procedure & & & .38 \\
\hline Lobectomy & $74(76.3 \%)$ & $83(85.6 \%)$ & \\
\hline Segmentectomy & $7(7.2 \%)$ & $8(8.2 \%)$ & \\
\hline Wedge resection & $16(16.5 \%)$ & $6(6.2 \%)$ & \\
\hline
\end{tabular}

SUVmax, Maximum standardized uptake value; N/A, not applicable.

TABLE 3. Comparison of solid and mixed tumor characteristics in patients matched for maximum standardized uptake value

\begin{tabular}{|c|c|c|c|}
\hline & $\begin{array}{l}\text { Solid tumor } \\
\quad(n=96)\end{array}$ & $\begin{array}{l}\text { Mixed tumor } \\
\quad(n=96)\end{array}$ & $P$ \\
\hline Age (y) & $65.4 \pm 10.4$ & $65.5 \pm 9.3$ & .94 \\
\hline Sex & & & .26 \\
\hline Male & 49 & 40 & \\
\hline Female & 47 & 56 & \\
\hline Whole tumor size $(\mathrm{cm})$ & $2.0 \pm 0.6$ & $2.1 \pm 0.6$ & .24 \\
\hline Solid tumor size $(\mathrm{cm})$ & $2.0 \pm 0.6$ & $1.5 \pm 0.7$ & $<.001$ \\
\hline SUVmax & $4.0 \pm 2.6$ & $4.0 \pm 2.6$ & N/A \\
\hline Lymphatic invasion & & & .12 \\
\hline Negative & 65 & 74 & \\
\hline Positive & 31 & 22 & \\
\hline Vascular invasion & & & .47 \\
\hline Negative & 62 & 67 & \\
\hline Positive & 34 & 29 & \\
\hline Pleural invasion & & & .071 \\
\hline Negative & 70 & 81 & \\
\hline Positive & 26 & 15 & \\
\hline Lymph node metastasis & & & .54 \\
\hline Negative & 80 & 84 & \\
\hline Positive & 16 & 12 & \\
\hline Procedure & & & .50 \\
\hline Lobar resection & 77 & 73 & \\
\hline Segmentectomy & 6 & 15 & \\
\hline Wedge resection & 13 & 8 & \\
\hline
\end{tabular}

SUVmax, Maximum standardized uptake value; N/A, not applicable.
TABLE 4. Comparison between solid and mixed tumor characteristics in patients matched for solid component size and maximum standardized uptake value

\begin{tabular}{lccc}
\hline & $\begin{array}{c}\text { Solid tumor } \\
(\mathbf{n}=\mathbf{7 9})\end{array}$ & $\begin{array}{c}\text { Mixed tumor } \\
(\mathbf{n}=\mathbf{7 9})\end{array}$ & $\boldsymbol{P}$ \\
\hline Age (y) & $64.4 \pm 10.7$ & $66.0 \pm 8.9$ & .27 \\
Sex & & & .62 \\
$\quad$ Male & $37(46.8 \%)$ & $41(51.9 \%)$ & \\
$\quad$ Female & $42(53.2 \%)$ & $38(48.1 \%)$ & \\
Whole tumor size $(\mathrm{cm})$ & $1.8 \pm 0.5$ & $2.2 \pm 0.5$ & $<.001$ \\
Solid component size $(\mathrm{cm})$ & $1.8 \pm 0.5$ & $1.8 \pm 0.5$ & $\mathrm{~N} / \mathrm{A}$ \\
SUVmax & $3.7 \pm 2.4$ & $3.7 \pm 2.6$ & N/A \\
Lymphatic invasion & & & .31 \\
$\quad$ Negative & $53(67.1 \%)$ & $60(75.9 \%)$ & \\
$\quad$ Positive & $26(32.9 \%)$ & $19(24.1 \%)$ & \\
Vascular invasion & & & 1.0 \\
$\quad$ Negative & $56(70.9 \%)$ & $56(70.9 \%)$ & \\
$\quad$ Positive & $23(29.1 \%)$ & $23(29.1 \%)$ & \\
Pleural invasion & & & .71 \\
$\quad$ Negative & $62(78.5 \%)$ & $65(82.3 \%)$ & \\
$\quad$ Positive & $17(21.5 \%)$ & $14(17.7 \%)$ & \\
Lymph node metastasis & & & .80 \\
$\quad$ Negative & $67(84.8 \%)$ & $69(87.3 \%)$ & \\
$\quad$ Positive & $12(15.2 \%)$ & $10(12.7 \%)$ & \\
Procedure & & & .15 \\
$\quad$ Lobar resection & $61(77.2 \%)$ & $66(83.5 \%)$ & \\
$\quad$ Segmentectomy & $5(6.3 \%)$ & $8(10.1 \%)$ & \\
$\quad$ Wedge resection & $13(16.5 \%)$ & $5(6.3 \%)$ & \\
SUVmax, Maximum standardized uptake value; N/A, not applicable. & \\
& &
\end{tabular}

In a next step, we evaluated whether mixed tumors exhibited malignant behavior and prognosis similar to those of solid tumors after matching for solid component size and SUVmax. In this matched model, solid tumors and mixed tumors had similar clinical characteristics and DFS. As shown in Figure 2, tumors with equivalent solid component size and SUVmax had the same malignant behavior (eg, lymphatic invasion), regardless of type. The DFS of patients with solid and mixed tumors was also comparable after matching for solid component size and SUVmax. These findings indicate that solid tumors and mixed tumors show similar biological behavior and prognosis when both have the same solid component size on HRCT and the same SUVmax value on PET/CT. In other words, solid component size on HRCT and SUVmax on PET/CT are important factors for evaluating malignant behavior of clinical stage IA lung adenocarcinomas before surgery, and this is regardless of the GGO proportion. Solid and mixed lung adenocarcinoma tumors with low SUVmax reflect pathologic noninvasiveness and may be good candidates for sublobar resection. We have previously reported, in the same population who were evaluated in the current study, that tumors with SUVmax less than 1.5 were not associated with lymph node metastasis or recurrence, ${ }^{12,18}$ and we recommend that individuals with clinical stage IA lung adenocarcinomas with 


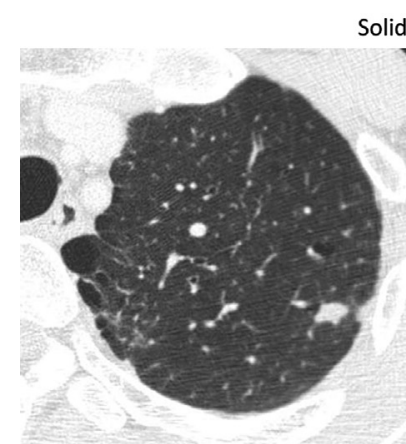

Whole tumor size $1.0 \mathrm{~cm}$

Solid component size $1.0 \mathrm{~cm}$

A

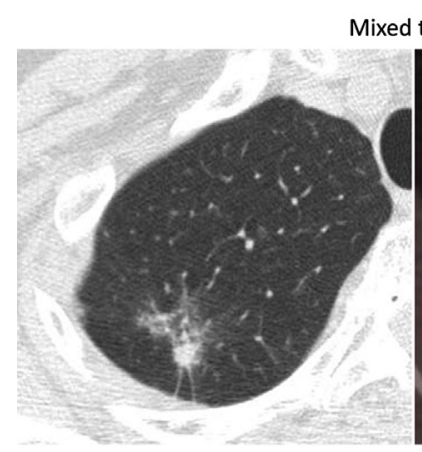

Whole tumor size $2.8 \mathrm{~cm}$

Solid component size $1.0 \mathrm{~cm}$

C

Negative for lymphatic invasion

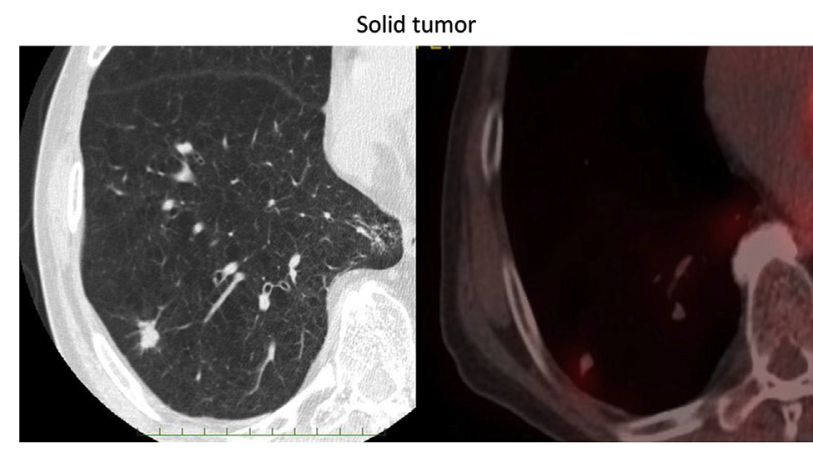

Whole tumor size $1.0 \mathrm{~cm}$

SUVmax 2.9

Solid component size $1.0 \mathrm{~cm}$

B

Positive for lymphatic invasion

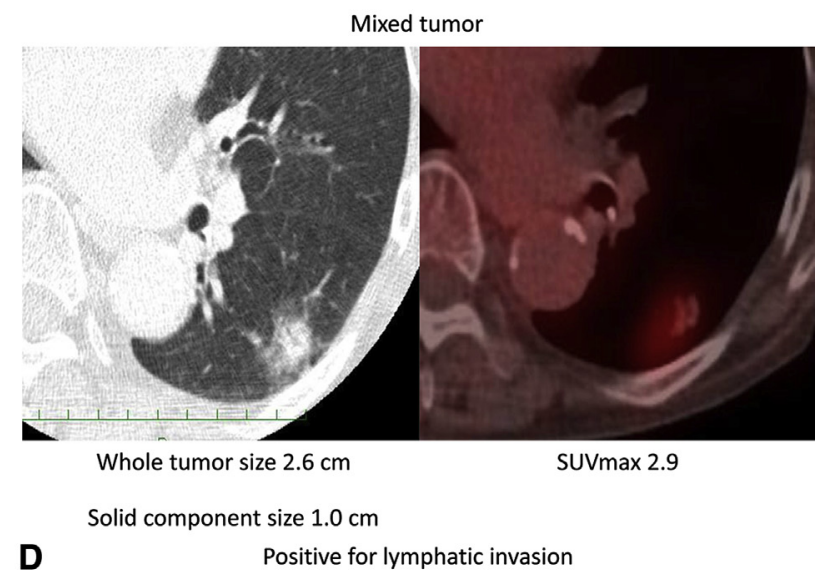

FIGURE 2. Examples of solid and mixed tumors on HRCT. A, Whole tumor size = solid component size: $1.0 \mathrm{~cm}$, SUVmax: 1.1 . This solid tumor was negative for lymphatic invasion. B, Whole tumor size = solid component size: $1.0 \mathrm{~cm}$, SUVmax: 2.9 . This solid tumor was positive for lymphatic invasion. C, Whole tumor size: $2.8 \mathrm{~cm}$, solid component size: $1.0 \mathrm{~cm}$, SUVmax: 1.1 . This mixed tumor was negative for lymphatic invasion. D, Whole tumor size: 2.6 $\mathrm{cm}$, solid component size: $1.0 \mathrm{~cm}$, SUVmax: 2.9. This mixed tumor was positive for lymphatic invasion. SUVmax, Maximum standardized uptake value.

SUVmax less than 1.5 should undergo sublobar resection with adequate surgical margins. ${ }^{18}$

One of the strengths of this study is the use of PET/CT in all patients. PET/CT, which is the diagnostic tool of choice for patients with non-small cell lung cancer, improves the sensitivity of preoperative staging and reduces the frequency of futile thoracotomies. ${ }^{19}$ In addition, SUVmax on PET/CT is a known prognostic factor for non-small cell lung cancer, especially for adenocarcinoma. ${ }^{6,11,12,16,17}$ For patients with clinical stage IA lung adenocarcinoma who do not undergo PET/CT, tumor type (solid or mixed) is an important factor for predicting malignant behavior and prognosis. Because the follow-up period was short in this study, long-term follow-up is needed to confirm the DFS results.

\section{CONCLUSIONS}

In cases of clinical stage IA lung adenocarcinoma, solid tumors are more malignant than mixed tumors even after matching for solid component size in both tumor types. However, solid tumors have the same malignant potential and prognosis as mixed tumors when both tumor types are matched for solid component size on HRCT and SUVmax on PET/CT.

\section{References}

1. Okada M, Koike T, Higashiyama M, Yamato Y, Kodama K, Tsubota N. Radical sublobar resection for small-sized non-small cell lung cancer: a multicenter study. J Thorac Cardiovasc Surg. 2006;132:769-75.

2. Nakayama H, Yamada K, Saito H, Oshita F, Ito H, Kameda Y, et al. Sublobar resection for patients with peripheral small adenocarcinomas of the lung: surgical outcome is associated with features on computed tomographic imaging. Ann Thorac Surg. 2007;84:1675-9.

3. National Lung Screening Trial Research Team, Aberle DR, Adams AM, Berg CD, Black WC, Clapp JD, Fagerstrom RM, et al. Reduced lung-cancer mortality with low-dose computed tomographic screening. N Engl J Med. 2011;365: 395-409.

4. Nakata M, Saeki H, Takata I, Segawa Y, Mogami H, Mandai K, et al. Focal ground-glass opacity detected by low-dose helical CT. Chest. 2002;121:1464-7.

5. Jang HJ, Lee KS, Kwon OJ, Rhee CH, Shim YM, Han J. Bronchioloalveolar carcinoma: focal area of ground-glass attenuation at thin-section CT as an early sign. Radiology. 1996;199:485-8. 
6. Tsutani Y, Miyata Y, Nakayama H, Okumura S, Adachi S, Yoshimura M, et al. Prognostic significance of using solid versus whole tumor size on highresolution computed tomography for predicting the pathological malignant grade of tumors in clinical stage IA lung adenocarcinoma: a multicenter study. J Thorac Cardiovasc Surg. 2012;143:607-12.

7. Goldstraw P, Crowley J, Chansky K, Giroux DJ, Groome PA, Rami-Porta R, et al. International Association for the Study of Lung Cancer International Staging Committee; Participating Institutions. The IASLC Lung Cancer Staging Project: proposals for the revision of the TNM stage groupings in the forthcoming (seventh) edition of the TNM Classification of Malignant Tumours. J Thorac Oncol. 2007;2:706-14.

8. Suzuki K, Asamura H, Kusumoto M, Kondo H, Tsuchiya R. "Early" peripheral lung cancer: prognostic significance of ground glass opacity on thin-section computed tomographic scan. Ann Thorac Surg. 2002;74:1635-9.

9. Nakamura H, Saji H, Ogata A, Saijo T, Okada S, Kato H. Lung cancer patients showing pure ground-glass opacity on computed tomography are good candidates for wedge resection. Lung Cancer. 2004;44:61-8.

10. Delbeke D, Coleman RE, Guiberteau MJ, Brown ML, Royal HD, Siegel BA, et al. Procedure guideline for tumor imaging with 18F-FDG PET/CT 1.0. J Nucl Med. 2006;47:885-95.

11. Nakayama H, Okumura S, Daisaki H, Kato Y, Uehara H, Adachi S, et al. Value of integrated positron emission tomography revised using a phantom study to evaluate malignancy grade of lung adenocarcinoma. Cancer. 2010;116:3170-7.

12. Okada M, Nakayama H, Okumura S, Daisaki H, Adachi S, Yoshimura M, et al. Multicenter analysis of high-resolution computed tomography and positron emission tomography/computed tomography findings to choose therapeutic strategies for clinical stage IA lung adenocarcinoma. J Thorac Cardiovasc Surg. 2011;141:1384-91.

13. Inoue M, Minami M, Sawabata N, Utsumi T, Kadota Y, Shigemura N, et al. Clinical outcome of resected solid-type small-sized c-stage IA non-small cell lung cancer. Eur J Cardiothorac Surg. 2010;37:1445-9.

14. Suzuki K, Kusumoto M, Watanabe S, Tsuchiya R, Asamura H. Radiologic classification of small adenocarcinoma of the lung: radiologic-pathologic correlation and its prognostic impact. Ann Thorac Surg. 2006;81:413-9.

15. Cerfolio RJ, Bryant AS, Ohja B, Bartolucci AA. The maximum standardized uptake values on positron emission tomography of a non-small cell lung cancer predict stage, recurrence, and survival. J Thorac Cardiovasc Surg. 2005;130:151-9.

16. Okada M, Tauchi S, Iwanaga K, Mimura T, Kitamura Y, Watanabe H, et al. Associations among bronchioloalveolar carcinoma components, positron emission tomographic and computed tomographic findings, and malignant behavior in small lung adenocarcinomas. J Thorac Cardiovasc Surg. 2007;133:1448-54.

17. Tsutani Y, Miyata Y, Misumi K, Ikeda T, Mimura T, Hihara J, et al. Difference in prognostic significance of maximum standardized uptake value on [18F]-fluoro2-deoxyglucose positron emission tomography between adenocarcinoma and squamous cell carcinoma of the lung. Jpn J Clin Oncol. 2011;41:890-6.

18. Tsutani Y, Miyata Y, Nakayama H, Okumura S, Adachi S, Yoshimura M, et al. Prediction of pathological node-negative clinical stage IA lung adenocarcinoma for optimal candidates undergoing sublobar resection. J Thorac Cardiovasc Surg. 2012;144:1365-71.

19. Fischer B, Lassen U, Mortensen J, Larsen S, Loft A, Bertelsen A, et al. Preoperative staging of lung cancer with combined PET-CT. N Engl J Med. 2009;361: 32-9. 